Es erscheint höchst zweifelhaft, ob diese Festlegungen des Gerichts als angemessen bezeichnet und auch ob sie in dieser, derart apodiktisch gefaßten Form werden beibehalten werden können ${ }^{47}$.

Vermutlich werden aber, namentlich wenn man amerikanische Erfahrungen nach hier überträgt, die Fragen nach dem Umgang mit unterschiedlichen Wertvorstellungen an die Schule in Zukunft deutlich radikaler gestellt werden; das bisherige, auf Integration über die Anerkennung von Gleichwertigkeit und Toleranz ausgerichtete Schulsystem wird sich möglicherweise verstärkt separierenden Entwicklungen gegenübersehen. Dabei könnte die geltende Verfassung das Einfallstor für gesellschaftliche Separierungstendenzen insoweit sein, als vorhandene, aber bezogen auf das gesamte Schulsystem in der Praxis eher unbedeutenden Möglichkeiten ${ }^{4^{8}}$ verfassungsrechtlicher Art dann eine erhöhte Wichtigkeit zukommt ${ }^{49}$ : Art. 7 Abs. 4 GG sieht das generelle Recht zur Errichtung privater Schulen vor; und von Art. 7 Abs. 5 GG wird das seit der Weimarer Verfassung geltende Prinzip der (sozialen) Integration durch die "für alle gemeinsame Grundschule « (Art. I 46 Abs. I Satz 2 WRV) ${ }^{\text {s。 }}$ in der Weise durchbrochen, daß private »Volksschulen«, d.h. in der heutigen Lesart Grundschulen und Hauptschulen ${ }^{51}$, auf Antrag von Erziehungsberechtigten begründet werden können, wenn diese dies aus Gründen ihrer besonderen Wertvorstellungen begehren und diese privaten Schulen dann als Weltanschauungs- oder Bekenntnisschulen geführt werden sollen ${ }^{52}$. Zwar gilt auch für derartige Schulen der Grundsatz der staatlichen Schulaufsicht nach Art. 7 Abs. I GG, zwar vermag auch der Staat die Einhaltung der allgemein geltenden Lernziele zu überwachen"3, aber mit der Errichtung entsprechender Schulen wird notwendig ein Schritt zur (sozialen) Separierung vollzogen. Eine solche Entwicklung des Schulwesens löst Probleme bedeutet dies, daß man sie zugleich befürworten soll?

\title{
Hans J. Kleinsteuber Selbstinszenierung eines Gerichts - Der U.S. Supreme Court
}

Kürzlich besuchte ich Karlsruhe, eine beschauliche Barockstadt, fernab der Hektik unserer Großstädte, die Stadt der Obersten Bundesgerichte. Das Verfassungsgericht ist mit Recht stolz auf seine luftige, offene Architektur am Rande des Schloßparks. Zugänglich wird es dadurch noch nicht, und mit Maschinenpistolen gerüstete

47 Ein möglicher Prüfstein hierfür könnte bereits in nächster Zeit der Antrag einer muslimischen Schülerin sein, die unter Hinweis auf die Tatsache, daß der Religionsstifter Mohammed selbst nie Sport getrieben habe und sie insoweit seinem Vorbild entsprechend leben wolle, die Befreiung vom Sportunterricht begehrt. Das VG Freiburg (Anm, 21) hat diesem Antrag stattgegeben, uber das Rechtsmittel gegen dieses Urteil ist noch nicht entschieden.

${ }_{4} 8$ Vogel, Zulassungsvoraussetzungen fur private Volksschulen, in: RdJB 1989, 299, 300; danach besuchten im Jahre 1987 nicht mehr als $1,1 \%$ aller Grundschüler/innen eine private Grundschule.

49 Ebenso Ladeur, Genehmigung privater Konfessionsschulen, in: RdJB I993, $282 \mathrm{ff}$.

so Vgl. Anschütz (Anm. 25), Art. 146, Anm. 4.

II Dazu auch Vogel, Das Recht der Schulen und Heime in freier Trägerschaft, 2. Aufl. Neuwied 1991, S. 19 m.w.N.

ऽ2 Ähnlich auch Richter, Verfassungsfragen multikultureller Gesellschaften, in: Gegenrede - Festschrift für Mahrenholz Baden-Baden 1994, S. 637 ff.; zur entsprechenden Situation etwa in Kanada vgl. Masemann, Canada: Ausschau nach pluralistischen Bildungsformen, in: RdJB 1993, 248, 253.

53 Worauf das Bundesverwaltungsgericht in seiner Entscheidung hinweist, a. a. O. (Anm. 2), s81 - unter Bezugnahme auf BVerwGE 90, 1 -, vgl. auch Ladeur (Anm. 49), $284 \mathrm{ff}$. 
Grenzschützer unterstreichen seine Exklusivität. Der Besuch des Bundesverfassungsgerichts muß vierzehn Tage vorher angemeldet werden - zur Sicherheitsüberprüfung. Ein Schockerlebnis hat die Stadt noch für den neugierigen Touristen: Der mit Stacheldraht und Fernsehkameras bis zur Unkenntlichkeit verbarrikadierte Bundesgerichtshof, mit den nach der Vereinigung wohl bestgeschützten Zäunen und Mauern der Republik. Die architektonische Gestaltung politischer Herrschaft hat bekanntlich immer etwas mit den Inhalten zu tun. Und daß besagter Bundesgerichtshof sich auch sonst nicht gerade offen gibt, erweist etwa die derzeitige Diskussion um die senatsinterne Geschäftsverteilung.

Wahrscheinlich meinen wir nach vielen Jahren der Gewohnheit, das müsse so sein. Da vermögen komparative Sichtweisen auf die Sprünge zu helfen. Wie gehen andere Demokraten mit ihrem Obersten Gericht um? Ein Szenario: Man stelle sich ein oberstes Bundesgericht vor, das sich aktiv seiner Außendarstellung widmet, das sich Transparenz als oberstes Ziel setzt und das den Bürger herzlich einlädt, an seinen Sitzungen teilzunehmen. Und wenn dieser immer noch zu wenig Interesse zeigt, so ködert man ihn mit einer beträchtlichen Dosis Entertainment. Das Gericht legt es regelrecht darauf an, eine Touristenattraktion zu werden. Es bietet gleichermaßen gelehrte wie unterhaltsame Führung durch das Gebäude, natürlich einschließlich seinem $>$ Allerheiligsten`, dem Gerichtssaal.

Öffnen wir das Restaurant für jeden, den das Innenleben der Justiz hungrig gemacht hat. Richten wir einen Kinoraum ein, in dem Videos über Geschichte und Arbeitsalltag des Gerichts informieren. Ein angeschlossenes Museum, in dem die Richter ganz menschlich mit shuman touch v vorgestellt werden, mit Herkunft und Familie, ja sogar mit der Schulbank, die sie in der Grundschule drückten. Leiten wir den Besucher zu einer Ausstellung, in der Karikaturen zur Justiz ausgehängt werden, viele davon vom bissigsten, was die Zeichnerzunft hervorgebracht hat. Zum Abschluß unseres Gedankenspiels wartet ein Geschenkeladen, nicht nur mit ernstem Stoff über die Bedeutung der Justiz, sondern auch mit Postkarten, ja Kaffeetassen und Whiskygläsern mit dem Emblem des Hohen Gerichts darauf. Oder wie wäre es mit einem schicken Schlüsselanhänger samt Justitia? Und natürlich wird eine Kopie des Hämmerchens als Souvenir angeboten, mit dem der Gerichtsvorsitzende Ruhe gebietet.

Dies Szenario klingt für deutsche Juristenohren grotesk. Aber dieses Gericht gibt es wirklich. Es handelt sich um nichts geringeres als das älteste Verfassungsgericht der Welt, den Supreme Court der USA. Dieses oberste Bundesgericht ist von unglaublicher Machtfülle, vereint es doch im wesentlichen die Funktionen unserer beiden Karlsruher Höfe, dem Bundesverfassungsgericht und dem Bundesgerichtshof, in einem Hause.

Heute residiert der Supreme Court in einem schneeweißen Tempel, hinter dessen Säulenreihen der Europäer wohl eher eine Kirche oder vielleicht noch einen Bahnhof vermuten würde. In der längsten Zeit des Gerichts bestand freilich keine Chance, eine derartige Show abzuziehen. Bis 1935 war es Untermieter im Kongreß und nutzte dort lange den alten Senatssaal. Den Bau eines eigenen Gehäuses betrieb sein Chefrichter W. H. Taft, der zuvor als (siebenundzwanzigster) Präsident der USA gedient hatte. Der Grundstein des heutigen Supreme Court wurde im Oktober 1932 gelegt, also mitten in der Weltwirtschaftskrise. Der Kongreß hatte beachtliche 9,74 Mio. Dollar zur Verfügung gestellt. Zur etwas selbstgerechten Folklore des Gebäudes zählt, daß die damals Verantwortlichen nicht nur mit der zugeteilten Summe auskamen, sondern noch 93 532,02 Dollars zurückgeben konnten.

Washington ist eine von Anbeginn geplante Hauptstadt, in der das politische System seinen steinernen Ausdruck in einer herrschaftlichen Architektur finden sollte. Die 
Idee der Gewaltenteilung lebt in der räumlich deutlich getrennten Ansiedlung des Hauptsitzes zweier Gewalten, Legislative und Exekutive, fort: Capitol und Weißes Haus. Beide verbindet symbolisch die Pennsylvania Avenue mit ihren vielen Ministerien, die frisch augurierte Präsidenten oder auch Astronauten im Triumphzug entlangzuziehen pflegen. In dieser Selbstinszenierung einer Kapitale hatte der Supreme Court anfangs keinen Stellenwert, der Planer von Washington, der Franzose L'Enfant, hatte ihn am Ausgang des I 8. Jahrhunderts schlicht vergessen. So mußte er als Grundstück nehmen, was im 20.Jahrhundert noch übriggeblieben war: Vom Stadtzentrum aus gesehen der Block links hinter dem Capitol Hill, gleich neben der im Stile der italienischen Renaissance gehaltenen Library of Congress.

Wollte man mit einem neuerrichteten Supreme Court im Ensemble der Washingtoner Herrschafts- und Repräsentationsarchitektur noch mithalten, mußte man sich stilistisch etwas besonderes einfallen lassen. Das Ergebnis jahrelanger Planungen wurde einer der größten weißen Marmorpaläste der Welt - so heißt es zumindest. Genau genommen handelt es sich um ein eher durchschnittliches, für die Hauptstadt der USA keineswegs ungewöhnliches Bürogebäude, dem eine mächtige, wenn auch reichlich überflüssige griechisch-römische Tempelfassade vorgesetzt wurde. Immerhin fällt der Supreme Court so schon von weitem auf und zieht die Neugierde der nun wirklich verwöhnten Hauptstadt-Besucher auf sich, obwohl er doch mit anderen, ähnlich spektakulären Orten konkurrieren muß, etwa dem FBI, wo für den Touristen sogar regelmäßig geschossen wird.

Im Vorfeld des Gebäudes operieren die Polizisten einer speziellen Supreme CourtTruppe, dominierende Farben schwarz und gold, und dirigieren die täglichen Besuchermengen. Die meisten der jährlich über 800000 Besucher sind neugierige Touristen aus der Provinz oder Schulklassen mit ihrem Sozialkundelehrer. Fast wie Portiers weisen die Uniformierten freundlich den Weg. Nur wenn Gerichtsverhandlungen anstehen, sind Schaulustige unerwünscht. Allerdings erklären die schwarzgoldenen Tempelwärter, wie man in diesem Fall an den öffentlichen Sitzungen teilnehmen kann. Gerichtstermine beginnen routinemäßig Montags um zehn Uhr, und nur zeitiges Kommen sichert einen der ca. zweihundert Sitzplätze im Gerichtssaal. Vergeben werden sie nach dem First-Come-First-Serve-Prinzip.

Ein wesentlicher Bestandteil der Selbstinszenierung des Gerichts sind die Touristenführer, die sich halb als Rechtsdozenten, halb als Animateure verstehen. Sie rekrutieren sich aus der Gruppe der Law Clerks, frischgebackenen Absolventen der besten Law Schools des Landes. Bei der Art, wie diese selbstbewußten Angehörigen einer zukünftigen Elite die eher provinziellen Besucher mit jovialer Geste durch die heiligen Hallen führt, wird viel von der Doppelbödigkeit der US-Demokratie zwischen führenden Machteliten und eher apathischen Massen sichtbar. Aber wie selbstverständlich öffnen sie allen Bürgern die Tore, freuen sich über deren Interesse und beantworten bereitwillig noch die ignoranteste Frage.

Wer den großen Platz vor dem Gebäude durchmessen hat, den führt eine riesige Freitreppe hinauf zum antiken Portal. Jeder Besucher des Gerichts kennt diese Tempelkulisse bereits aus dem Fernsehen, stellt sie doch den typischen Hintergrund für die Gerichtskorrespondenten dar. Dann tritt er durch eine der gigantischen bronzenen Eingangstüren, das Stück mit sechseinhalb US-Tonnen Gewicht. Ihre acht Reliefs sollen das Wachstum des Rechts von den Römern bis zu den Amerikanern versinnbildlichen.

Natürlich wird der Besucher erst einmal durch eine elektronische Waffenkontrolle geschleust, ähnlich der auf US-Flughäfen. Das dauert pro Person einige Sekunden. Mehr ist angesichts der Massen von Neugierigen auch gar nicht möglich. Darauf kann er sich entweder in den öffentlichen Teilen des Gebäudes frei bewegen oder sich 
einer Führung anschließen, die in das Heiligste des Tempels, den Gerichtssaal, führt. Sicherheitsprobleme scheint es nicht zu geben, obwohl das Gebäude nur einige Blocks von einem der übelsten Slums der USA entfernt liegt.

Den Besucher erwartet eine riesige, kathedralhaft düstere Eingangshalle mit Säulen, Friesen und einer vielfarbig bunten Kassettendecke, von der gewaltige Lüster aus Gold und Glas herabhängen. Aus den Nischen der Wände blicken ernst die marmornen Büsten aller bisherigen Chefrichter auf die Eindringlinge, natürlich im römischen Stil gehalten.

Am Ende dieses Foyers, dort wo in den Römertempeln das Götterbild stand, finden wir das eigentliche Heiligtum, den Great Hall genannten Gerichtssaal.

Der ganze Bau scheint vor allem eine Botschaft zu atmen: Die USA stehen in der Tradition der großen Rechtskulturen, vorneweg der Antike und hier speziell der Römer. Das erscheint seltsam realitätsfern, denn die Rechtspraxis des angelsächsischen Common Law hat mit der kontinental-römischen des europäischen Festlandes wenig gemein. In der Law Bibliothek der Library of Congress gibt es z. B. Abteilungen für britisches, kanadisches und australisches Recht, der Rest der Welt wird unter >Foreign Law` geführt.

Die Richter sitzen, deutlich erhöht, an einem riesenhaften, leicht gekrümmten Tisch, reich beschnitzt, der aus nur einem Stück Tropenholz, einem honduranischen Mahagonibaum, hergestellt wurde. US-Fahnen links und rechts setzen die wohl unvermeidlichen patriotischen Akzente. Die Richter können es sich in großen, mit schwarzem Leder aufgepolsterten Sesseln bequem machen, die nach ihren persönlichen Vorgaben individuell angefertigt werden. Daher variieren sie in der Höhe, was dem sonst so ordentlichen Ensemble ein unruhiges, fast schlampiges Moment hinzufügt. Direkt unterhalb der Richter sitzen die wichtigen Teilnehmer des Verfahrens: Anwälte und Angestellte des Gerichts. Für besondere Zelebritäten wird edles Gestühl bereitgehalten, etwa für den amtierenden Präsidenten oder frühere Verfassungsrichter. Der Präsident der USA allerdings läßt sich höchst selten sehen, womit er demonstrativ die Autonomie der Dritten Gewalt achtet und respektiert.

Linkerhand befinden sich leuchtend rot gepolsterte Bänke für Pressevertreter, die bis heute mit Stift und Papier arbeiten müssen. Deshalb gibt es aus dem Gerichtssaal von den dort arbeitenden Richtern nur Zeichnungen, die - den räumlichen Gegebenheiten folgend - immer deren rechte Gesichtshälfte zeigen. Kameras sind nicht erlaubt. Als Begründung hört man, daß sich die höchsten Verfassungsschützer nicht ihre Urteile auf 30 Sekunden lange ,Sound Bites der Infotainment-Nachrichten im Fernsehen reduzieren lassen wollen. Gegenüber der Pressebank findet sich eine gleichfalls rote Sitzgelegenheit für eine ganz andere privilegierte Bevölkerungsgruppe, die Familienangehörigen der Richter. Schließlich gibt es, deutlich mit Messingbrüstungen abgesetzt, damit sie den Gralshütern nicht zu nahe kommen, noch die eher bescheidenen und unbequemen Bestuhlungen für die Zuhörer.

Zur Freude des Besuchers schaut bei allem Pathos und Ernst doch hier und dort der Schalk hinter den kühlen Säulen des Supreme-Court-Gebäudes hervor. So versteckt sich hinter der römischen Prachtarchitektur nicht nur ein Ensemble relativ normaler Büroräume, es findet sich auch ein sgymnasium‘, eine Art Turnhalle mit allen Einrichtungen dort, in der die Richter und ihre Mitarbeiter ein sworkout genießen können. Auch Friseur und Schneider haben sich in diesem Pseudo-Tempel mit merkantilen Nischen niedergelassen.

Die Neugier der Besuchermassen wird recht wirkungsvoll auf die umfangreiche Ausstellung im Keller geleitet, wo zwischen qualitätsvoller Bildung und einer Art Ahnenverehrung (hier sind tatsächlich Roben, Schulbänke und andere Reliquien früherer Richter ausgestellt) auch ausführlich über die Möglichkeiten des Impeach- 
ments, der Amtsenthebung von fehlenden Richtern durch den Kongreß informiert wird. Richtig komisch mutet die Karikaturensammlung an. Dort erkennt man Politiker, die ihnen hörige Richter lancieren. Richter drehen an einem Glücksrad, um die angeblichen Verfassungsgrenzen der Obszönität auszuloten - ein sowieso eher peinliches Kapitel amerikanischer Verfassungsrechtsprechung. Eine weitere Karikatur zeigt, was passiert, wenn das Fernsehen einmal den Gerichtssaal erobern sollte. Alle Richter grinsen in die Kamera, der Vorsitzende kämmt sich noch rasch verstohlen, ein Teleprompter gibt den Text vor: "Do you swear to tell the truth... « So ganz abwegig ist diese Vision nicht einmal, denn inzwischen ist in 45 Einzelstaaten der USA das Fernsehen erlaubt, und ein spezieller Kabelkanal Court TV zeigt die spannendsten Mord-, Vergewaltigungs- und Drogenverfahren.

Versuchte Einflußnahme und Kritik von Betroffenen gehören zum festen Bestandteil der Rechtspolitik in den USA: Sie hat auch architektonisch ihren natürlichen Ort gefunden, die große Plaza vor der imposanten Tempelfassade, die leicht einen Aufzug von einigen tausend Protestlern aufzunehmen vermag. Hier wird tatsächlich häufiger demonstriert, und niemand scheint diese Möglichkeit zu mißbrauchen. Vielleicht ist es gerade diese Offenheit, die alle Beteiligten die Spielregeln demokratischer Anständigkeit einhalten läßt. Auf dem Vorplatz werden auch die TV-Kameras aufgebaut, vor denen die Kontrahenten bei anstehenden Gerichtsverfahren ihre jeweiligen Positionen begründen.

Wer bleibende Erinnerungen in die Heimat mitnehmen will, muß dem hauseigenen Andenkenladen einen Besuch abstatten, wo eine reiche Sammlung von GerichtsNippes auf den Käufer wartet. Er sei dem deutschen Touristen anempfohlen, denn die knallbunten Bilderbücher und gerichtswappen-bewehrten Küchengegenstände schocken garantiert jeden daheimgebliebenen Juristen. Zum Abschluß locken Restaurant und Cafeteria. Im Restaurant werde man, so wird volksverbunden im Reiseführer schwadroniert, sogar echte Richter beim Tafeln beobachten können. Aber wie oft in den USA erweist sich auch hier die Bürgernähe eher als Illusion. Tatsächlich haben die Richter einen eigenen Speiseraum, in edlem, altem Sheraton Mahagoni gehalten und mit Orientteppichen ausstaffiert.

Lehren für die Bundesrepublik? Nun, die Verantwortlichen für unsere Obersten Bundesgerichte sollten nicht gleich einen professionellen Animateur der Disney Productions einfliegen lassen. Aber ein wenig mehr Bürgernähe ist augenscheinlich überfällig. Übrigens gibt es wenigstens einen Ansatz: Das von einem Pächter betriebene Casino des Bundesverfassungsgerichts am Rande des Komplexes ist aus dem Schutzbereich ausgeklammert und als einziges ohne Hindernis zugänglich - es bietet sich gar für Hochzeiten und andere Festlichkeiten an. Diese Räumlichkeiten, die heute noch den Charme einer echten Behördenkantine ausstrahlen, aber durchaus von einem Teil der Verfassungsrichter zur Essensaufnahme genutzt werden, sollen allerdings demnächst zu weiteren Dienstzimmern umgebaut werden ... 
Dagmar Schiek

\section{"Vereinbarkeit von Beruf und Familie« - vereinbar mit der arbeitsmarktlichen Gleichstellung von Frauen?}

Am I. September 1994 ist das Zweite Gleichberechtigungsgesetz in Kraft getreten ${ }^{\text {. }}$ Erklärtes Ziel des Gesetzesvorhabens ist es, "zusätzliche gesetzliche Regelungen (...) zur besseren Vereinbarkeit von Familie und Beruf « zu schaffen ${ }^{2}$. Auch die acht neueren Landesgleichstellungsgesetze ${ }^{3}$ enthalten Regelungen zur Vereinbarkeit von Beruf und Familie. Nur die Quotenvorschriften der beiden ältesten Frauenfördergesetze der Länder Nordrhein-Westfalen und Saarland 4 verzichten auf solches Beiwerk. Regelungen zur Förderung der besseren Vereinbarkeit von Familie und Beruf gelten manchem als wesentlich erfolgversprechenderes Konzept der Herstellung von Chancengleichheit zwischen Frauen und Männern als die Quotierungs und werden auch von Kritikern der Quote wärmstens empfohlen ${ }^{6}$. Das Thema der arbeitsrechtlichen Abteilung des 60. Juristentages "Welche Maßnahmen empfehlen sich, um die Vereinbarkeit von Berufstätigkeit und Familie zu verbessern? « wurde denn auch von beiden Gutachtern als ausgesprochenes Frauenthema verstanden. Soviel Einigkeit provoziert die Fragestellung, ob Vereinbarkeit, wie sie das gegenwärtige Arbeitsund Dienstrecht - auch nach der Änderung durch das Zweite Gleichberechtigungsgesetz - versteht, überhaupt etwas mit der Gleichstellung von Frauen und Männern am Arbeitsmarkt zu tun hat.

\section{A. „Vereinbarkeit von Beruf und Familie im geltenden Recht}

Die kontinuierliche Verantwortung für die alltägliche Betreuung von Kindern liegt von wenigen Ausnahmefällen abgesehen - bei Frauen. »Vereinbarkeitsregeln« sind deshalb vorrangig Frauenrecht. In der modischen Diskussion um »Vereinbarkeit« geht dieser Fakt häufig in geschlechtsneutralen Formulierungen unter. Geschlechtsspezifische Formulierungen aber, die an die bemerkenswert veränderungsresistente Realität anknüpfen, scheinen den Anspruch auf Veränderung dieser einseitig Frauen belastenden Arbeitsteilung aufzugeben - ein unlösbares Dilemma. Als unbefriedigender Kompromiß wird im folgenden von aktiven Eltern gesprochen, die zu 99,9\% Mütter sind - die Leserin und der Leser möge jeweils über das Wort »aktiv« stolpern

I Vom 21. 4. I994, BGBI. I, 1406

2 Vgl. Gesetzentwurf der Bundesregierung vom 21.7.1993, BT-Drs. 12/5468, unter A. Zielsetzung; Abg. Nolte (CDU), 222. Sitzung des Bundestages am 21.4.1994 (Zweite und Dritte Lesung des 2. GleibG), Stenographischer Bericht S. 19153 (B); Abg. Böhmer (CDU), ebenda, S. 19159 (D).

3 In der Reihenfolge des Inkrafttretens: Landesgleichstellungsgesetz Bremen vom 29. I I. 1990 (BremGVBI. S. 433); Berliner Landesgleichstellungsgesetz vom 31. 12. 1990 (BlnGVB1. 1991, 8), geändert durch Gesetz vom I 3. 4. 1993 (BlnGVBl. I 84); Hamburgisches Gleichstellungsgesetz vom 19.3. I 99 I (HambGVBl. 75, in Kraft seit 1.4.1992); Frauenfordergesetz des Landes Sachsen-Anhalt vom 27.6.1993 (GVBI. 762); Hessisches Gleichberechtigungsgesetz vom 13. I 2. 1993 (HessGVBl. 729); Gleichstellungsgesetz Mecklenburg-Vorpommern vom 14.3.1994 (MV GVBl. 343); Sächsisches Frauenfördergesetz vom 31.3. 1994 (SähsGVBI. 684); Niedersächsisches Gleichberechtigungsgesetz vom 16.6. 1994 (NdsGVBl. 246); Brandenburgisches Landesgleichstellungsgesetz vom 8.7.1994 (GVBI. Brdbg Teil I, 254).

4 Saarländisches Gesetz zur Förderung von Frauen und zur Änderung sonstiger dienstrechtlicher Vorschriften vom 6.7.1989, ABI. Saarl. S. 977, nordrhein-westfalisches Frauenfördergesetz vom 31. 10. 1989, GV. NW. S. 567 .

5 Battis, Eisenhard, ZRP 1994, 18 (20).

6 Ladeur, ZRP 1993, 47; Sachs, NJW 1988, 553 ( 556 liSp). 